\title{
AMDA 2.13: A major update for automated cross-platform microarray data analysis
}

\author{
Dimos Kapetis ${ }^{1,}$, Ferdinando Clarelli ${ }^{1}$, Federico Vitulli ${ }^{1}$, Nicole Kerlero de Rosbo ${ }^{2}$, Ottavio Beretta ${ }^{1}$, Maria \\ Foti $^{1,3}$, Paola Ricciardi-Castagnoli ${ }^{1, \#}$, and Francesca Zolezzi ${ }^{1, \#}$ \\ ${ }^{1}$ Genopolis - Consortium for Functional Genomics, Department of Biotechnology and Biosciences, University of \\ Milano-Bicocca, Piazza della Scienza 2, 20126 Milan, Italy, ${ }^{2}$ Fondazione IRCCS Istituto Neurologico Carlo \\ Besta, Via Celoria 11, Milan 20133, Italy, and ${ }^{3}$ University of Milano-Bicocca, Department of Biotechnology and \\ Biosciences, Piazza della Scienza 2, 20126 Milan, Italy
}

* Present address: Fondazione IRCCS Istituto Neurologico Carlo Besta, Via Celoria 11, Milan 20133, Italy

${ }^{\#}$ Present address: Singapore Immunology Network (SIgN), 8A Biomedical Grove, 138648, Singapore

BioTechniques 53:33-40 (July 2012) doi 10.2144/0000113889

Keywords: data analysis; microarrays; analytical pipelines; automation; open-source software

Supplementary material for this article is available at www.BioTechniques.com/article/113889

Microarray platforms require analytical pipelines with modules for data pre-processing including data normalization, statistical analysis for identification of differentially expressed genes, cluster analysis, and functional annotation. We previously developed the Automated Microarray Data Analysis (AMDA, version 2.3.5) pipeline to process Affymetrix 3' IVT GeneChips. The availability of newer technologies that demand open-source tools for microarray data analysis has impelled us to develop an updated multi-platform version, AMDA 2.13. It includes additional quality control metrics, annotation-driven (annotation grade of Affymetrix NetAffx) and signal-driven (Inter-Quartile Range) gene filtering, and approaches to experimental design. To enhance understanding of biological data, differentially expressed genes have been mapped into KEGG pathways. Finally, a more stable and user-friendly interface was designed to integrate the requirements for different platforms. AMDA 2.13 allows the analysis of Affymetrix (cartridges and plates) and whole transcript probe design (Gene 1.0/1.1 ST and Exon 1.0 ST GeneChips), Illumina Bead Arrays, and one-channel Agilent $4 \times 44$ arrays. Relative to early versions, it supports various experimental designs and delivers more insightful biological understanding and up-to-date annotations.

The main issues for commercially available microarray platforms such as Affymetrix, GeneChip, Illumina BeadChip, and Agilent arrays are pre-processing, statistical data analysis, and subsequent extraction of biological knowledge. Analysis of microarray data should follow four main steps: (i) quality control, (ii) data pre-processing, (iii) identification of differentially expressed genes (DEG), and (iv) extraction of biological knowledge from DEG. The modules for quality control and data normalization are the most important steps of array evaluation and are platform-dependent. Quality controls help the user detect array artifacts or outliers and evaluate the uniformity of experimental groups in such a way as to choose the most appropriate pre-processing steps. Subsequently, the identification of DEG, generally the main objective of a microarray experiment, requires the application of statistical tests. Finally, to get an overview of the biological phenomena underlying the experiment, the identified DEG are functionally enriched by the use of public domain annotation databases like Gene Ontology (1) or pathway analysis terms, such as the Kyoto Encyclopedia Gene and Genomes (KEGG) (2).

Currently, many commercial or free tools exist to perform the common steps described above, such as the Automated Microarray Data Analysis [AMDA, version 2.3.5; (3)] pipeline that we developed to process Affymetrix 3' IVT GeneChips. However, few such tools provide the required modules for multiplatform analysis. Well-known web applications such as Gene Expression Pattern
Analysis Suite (4), Gene'TrailExpress (5), Taverna (6), GenePattern (7), or Galaxy (8) implement many tools for microarray or genomic analyses and for meta-analysis, but do not offer pre-processing for multiplatform analysis. Others, like Integrative Array Analyzer (9) and Expression Profiler (10), provide a user-friendly interface for different microarray platforms, but lack the pre-processing normalization and quality control steps.

Several solutions for pre-processing steps are available in the OneChannelGUI Bioconductor package (11), Alt Analyze (12), or ArrayMining web application (13). However, while some of these cover all the steps of data analysis and array platforms, none provides a comprehensive explanatory report of the analyzed data. 
Here, we report an updated multiplatform version of AMDA, AMDA 2.13, which performs a fully automated microarray analysis for Affymetrix GeneChips, Illumina BeadChip, and Agilent one-color $4 \times 44$ gene expression platforms. AMDA 2.13 has been implemented for R-2.14 or higher; it includes all the major data analysis steps as well as up-to-date annotations.

\section{Materials and methods}

\section{Hard-drive Space, Processor,} and Memory Requirements

AMDA 2.13 is freely available for Linux, Windows, and Mac OS X operating systems (https://sourceforge.net/projects/automicroarray/files/). The software requires a minimum of $300 \mathrm{MB}$ of hard-drive space for installation of additional packages and annotation data; a minimum of $2 \mathrm{~GB}$ of RAM and Intel Pentium III processor speed for Affymetrix 3' IVT array analysis; an additional free hard-drive space of around $100 \mathrm{MB}$ for writing the output files; and additional RAM (up to $4 \mathrm{~GB}$ ) and harddrive space (up to $5 \mathrm{~GB}$ free) recommended for large numbers of arrays, when using Gene 1.0/1.1 ST and Exon 1.0 ST GeneChips.

\section{Updated Interface}

The functions with command-line options are called through a GUI interface. The previous $\mathrm{tcl} / \mathrm{tk}$ Widget interface was substituted with gWidget package of the CRAN repository (http://cran.r-project.org/).

\section{Data Pre-Processing}

AMDA 2.13 accepts BeadStudio version 3.0 software output and pre-processes Illumina BeadChips through lumi (14). Affymetrix Gene 1.0/1.1 ST and Exon 1.0 ST .CEL files can be imported and normalized with Oligo (14). The summarization step for Gene 1.0/1.1 ST and Exon 1.0 ST arrays is computed at transcript cluster level. Agilent $4 \times 44$ gene expression files exported by Agilent Feature Extraction (AFE) 9.1.3.1 (or later version) image analysis software can be read and analyzed though Agi4×44PreProcess (14). The latter package offers a number of different pre-processing steps: background correction and normalization between samples, filtering probes by quality flags, and summarization of replicated probes. The user can choose between the foreground signal (gProcessedSignal or gMeanSignal) and the background signal for the background correction (gBGMedianSignal or gBGUsed). The user also decides the methods for background correction ("none," "half," "normexp") and normalization between arrays ("none," "quantile," "vsn”). AFE provides a flag for each spot, which identifies different quantification errors of the signal. Quality filters are also implemented to remove controls, well-above background, well-above negative controls, saturation, population outliers, non-uniform outliers, and non-available values. Negative controls are removed by default from the ExpressionSet (ES). Additional normalization methods, such as frozen RMA (fRMA) and VSN, are available for $3^{\prime}$ IVT through Bioconductor (14) and have been implemented. fRMA algorithm is available for HG-U133A and HG-U133Aplus2.0 Affymetrix GeneChips. Pre-processing and annotation steps are also implemented for not widely used arrays such as Arabidopsis thaliana and Saccharomyces cerevisiae.

Batch effect correction: The importance of compensating for non-biological batch variation has been extensively documented recently (15). In order to reduce variations in experimental effects or batch effects, thereby empowering the detection of DEG through our pipeline, we have introduced empirical Bayesian Methods with ComBat (16).

Quality control metrics among arrays: Affymetrix Relative Log Expression (RLE) and Normalized Un-scaled Standard Error (NUSE) are calculated and plotted according to affyPLM, Oligo, or Agi4x44PreProcess Bioconductor packages (14). In order to assess quality of replicates and evaluate relations between classes after DEG selection, Principal Component Analysis (PCA) was added. PCA reduces data dimensionality through linear combinations of variables according to a criterion of variance maximization, thus creating new latent variables (17). The first three principal components are computed and plotted with respect to each other in the three possible combinations, together with relative histograms of explained variances. The hierarchical clustering among arrays is calculated and plotted with Easy Microarray Analysis package (http://cran.r-project.org/), with agglomerative average-linkage hierarchical-clustering algorithm as default. Pearson correlation was chosen as default similarity measure.

Inter-Quartile Range (IQR) and Affymetrix NetAffx annotation grade filter wrapper function: To address the well-known multiple-testing problem in microarray data analysis, a set of non-specific filtering functions was provided through genefilter (14) implemented in the IQR wrapper function (IQRFilter), which provides a measure of the variability of each probeset signal across the given data set.

The IQRFilter calculates an IQR for each probeset across all samples, and proceeds in two different ways: (i) if the IQR value is equal to "auto," the function evaluates the optimal
A

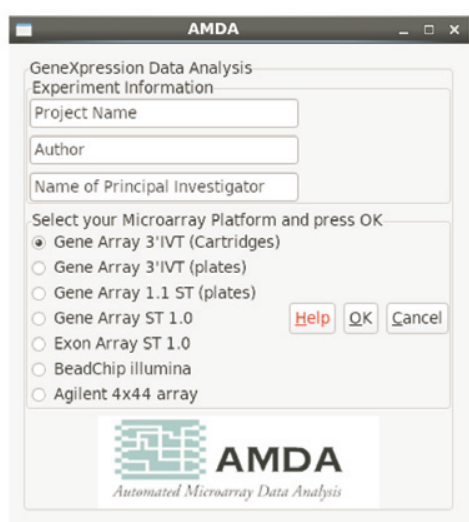

B

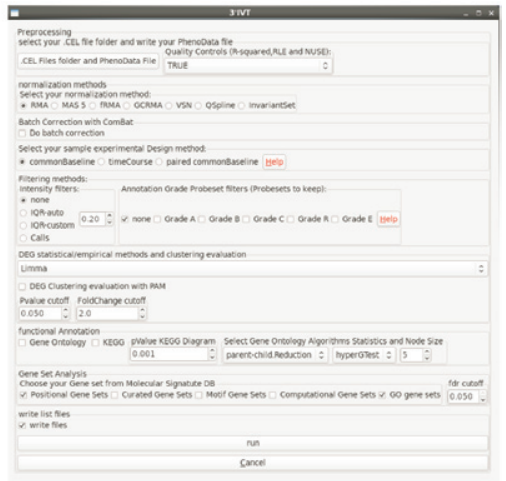

Figure 1. The gWidget AMDA interface. Summary of the most important two gWidgets required to run a complete analysis. They also allow the tuning of almost all the parameters of the tools available in the pipeline. The gWidgets cover the choice of the platform and experiment information (A), and of the loading and preprocessing of data, experimental design, filtering methods, functional evaluation, and setting the options for writing gene lists (B).

IQR value and provides a figure describing the IQR profile, a data set before and after filtering, and the IQR value automatically selected; and (ii) the IQR value can be a userdefined value (IQR value $>0$ ).

Optimal IQR threshold evaluation is performed to identify probesets with the highest variability in signal across arrays. In particular, a 100-point vector (at a step $\mathrm{h}=0.01)$ is created to compute and plot an IQR profile for each vector; the first derivative of such profile is then computed and its minimum point is identified (i.e., that corresponding to the steepest slope of IQR plot). This will be the point of maximum variation of the data set. A check for convergence on the estimated minimum (IQR opt) is then performed, by progressively reducing the step (thus dividing h by 0.1 at each iteration, $I Q R \_o p t-h<I Q R \_o p t<$ $\left.I Q R \_o p t+h\right)$, in order to refine the search for minimum value. 


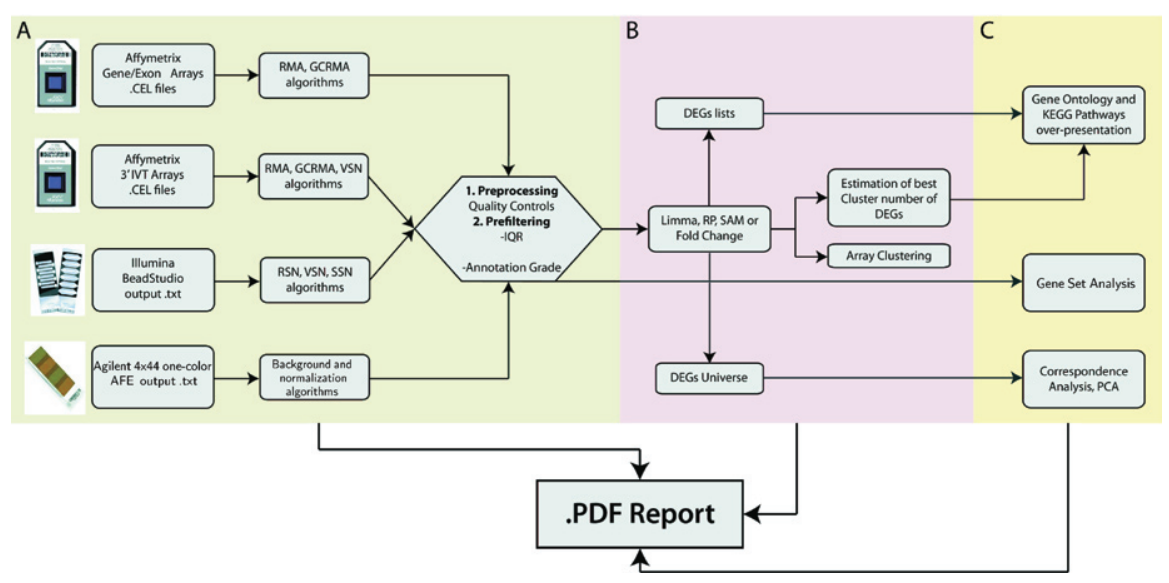

Figure 2. Overview of the AMDA pipeline. Summary of the most important features of the AMDA pipeline. Black arrows show the flow of information from the raw data to the three main types of analysis: Data loading and preprocessing ( $A$, green background), DEG selection and clustering (B, pink background), and functional classification (C, yellow background).

A

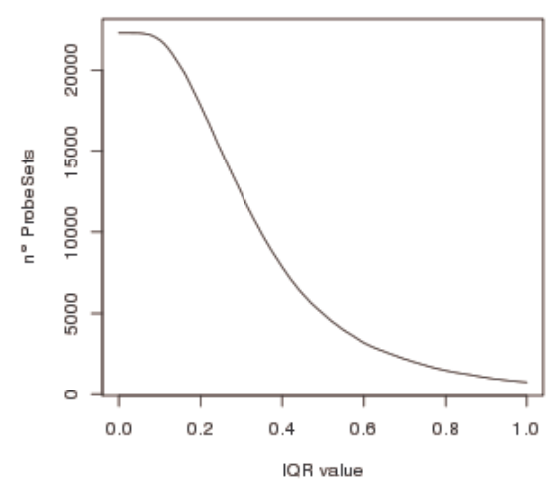

B

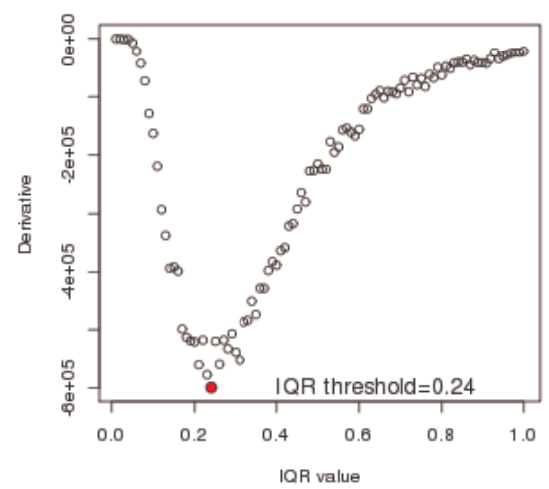

C

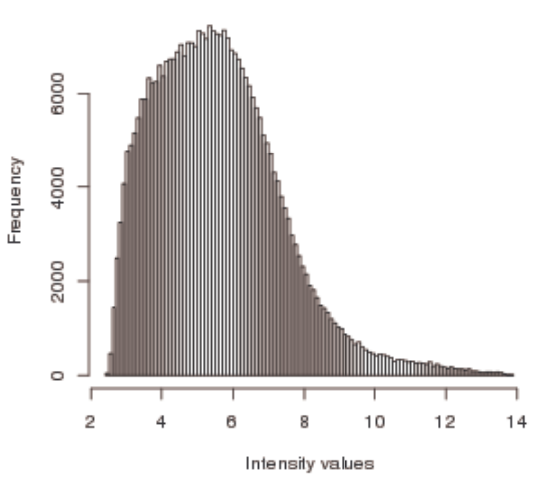

D

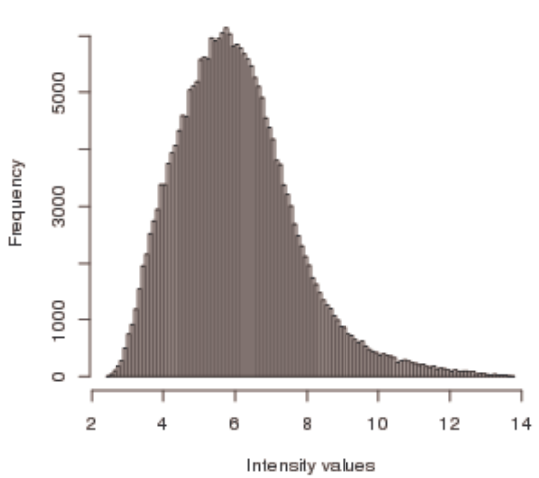

Figure 3. IQR function for gene selection. (A) IQR profile showing the number of probesets related to each IQR value. When IQR is 1 , no probeset passes the threshold, whereas when IQR value is 0 all probesets pass the selection. The distribution of these points is sigmoidal. (B) Plot of the values of the first derivatives of the IQR profile, with the minimal value being the inflection point where variability is maximal. This value is chosen for filtering the ES. (C) and (D) show the data set before and after filtering, respectively.

Affymetrix NetAffx Analysis Center (www.affymetrix.com/analysis/index. affx) records five levels of relationships (Grades A, B, C, R, or E) between 3' IVT probesets and the current transcript record. Each grade assignment provides information between probeset and pair-wise genomic alignment. The GradeSelection function using NetAffx annotation permits selection of the annotation grade record at the pre-processing stage.

Oligo package selects between probe cross-hybridization by selecting different probe groups divided in three categories, as follows: (i) core (Gene 1.0/1.1 ST and Exon 1.0 ST): probesets supported by RefSeq and full-length GeneBank transcripts with "unique" and "mixed" targeting genome positions; (ii) extended (Exon 1.0 ST): probesets with cDNA support information; and (iii) full (Exon 1.0 ST): probesets supported by gene predictions.

Wrapper Functions for DEG Selection: limma-paired Sample and RankProduct The limma test provided by AMDA 2.3.5 worked on the so-called "commonBaseline" experimental design for DEG identification, allowing comparison of samples to a common reference. Limma has been extended to the paired case, e.g., in cases where for each individual in a group, the same sample undergoes two different treatments. By using a paired $t$-test, we take pairing into account thus decreasing the variance of our estimates and eliminating spurious sources of variations such as sex, age, etc. The LimmaPairedSamples function applies the limma linear model (18) to identify the set of common DEG characteristic for a treatment group as compared with a control group. The RankProduct (RP) method (19), a powerful method for data set with limited sample size, was also integrated for two classes using the Rankprod package of Bioconductor (14).

\section{Venn Diagrams}

For multiple comparisons between conditions, Venn diagrams are produced to visualize mutual intersections of up to three different lists of DEG in condition contrasts. An image reporting the number of DEG with respect to all possible intersections of categories is plotted, and a set of tab-delimited text files of the annotated DEG is provided.

\section{Gene Set Analysis}

Single-gene statistical approaches for DEG identification in AMDA 2.3.5 is affected by major limitations such as statistical thresholds applied. Gene effects on pathways could therefore be missed when a single-gene statistical test is applied.

To improve the functional meaning of the DEG, Gene Set Analysis (GSA; http:// www-stat.stanford.edu/ tibs/GSA/) was added to AMDA 2.13. GSA aggregates functionally related genes using different resources such as biological pathways and chromosomal bands in relation to similar 


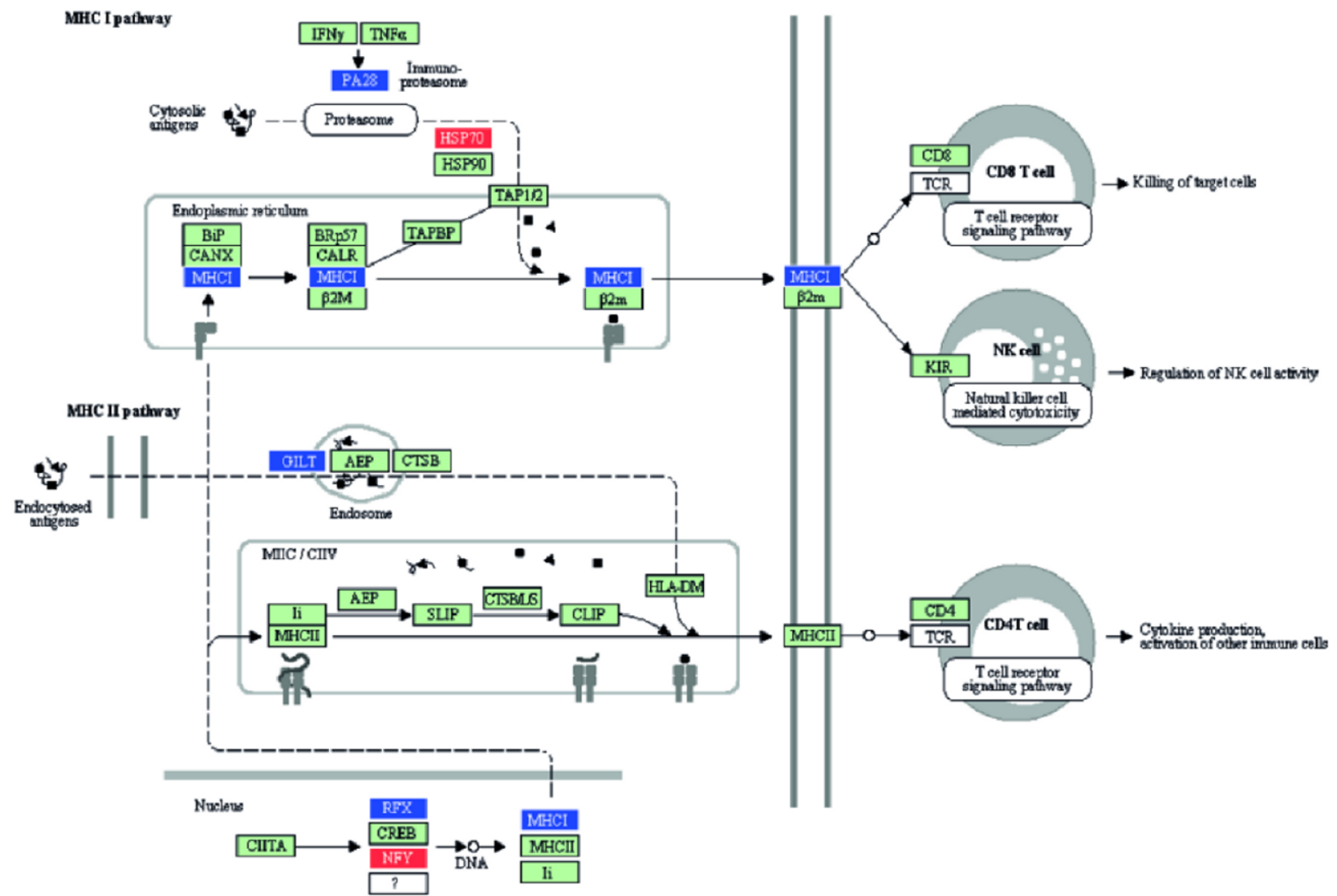

Figure 4. KEGG diagram of the pathway. Down-regulated genes are represented by blue boxes and up-regulated genes are represented by red boxes. The topology of mapped DEG facilitates the selection of genes of interest in a specific pathway.

gene expression patterns. GSA was implemented for additional gene-set collections to identify DEG that share the same biological context. GSA allows the analysis of more comprehensive gene-set collections for overrepresentation; it uses the maxmean statistic and restandardization process to summarize gene sets. AMDA 2.13 uses a total of 6,769 gene sets of the Molecular Signatures Database (MSigDB 3.0, www.broadinstitute.org/gsea/ msigdb), which are divided into five major collections (positional, curated, motif, computational, and gene ontology gene sets).

\section{Gene Ontology Functional}

Enrichment of DEG

With AMDA 2.3.5, enrichment analysis of DEG through Gene Ontology (GO) and KEGG pathways was performed using the hyper-geometric distribution through GOStats to assess over-representation of DEG in functional terms (3). The implementation of TopGO (20) on AMDA 2.13 integrates many algorithms and statistical tests for GO term enrichment analysis. It includes new statistical tests and new algorithms (such as eliminating genes, weighting genes, and parent-child relationships) that deal with GO graph structure, giving better understanding of the relationship between GO terms for calculating statistical significance (20).

\section{Graphical Representation}

of KEGG Pathways

DEG can be mapped into KEGG diagrams through Entrez Gene identifier (21). KEGGSOAP Bioconductor package was used to highlight DEG on KEGG pathways. The latest release of KEGG pathways, dated 15 March 2011, was based on mappings from KEGG GENOME ftp://ftp.genome. $\mathrm{jp} / \mathrm{pub} / \mathrm{kegg} / \mathrm{genomes}$.

\section{Results and discussion}

As described in our previous work (3), AMDA 2.3.5 was entirely written in $\mathrm{R}$ language making intensive use of Bioconductor classes (such as ES, Affybatch, AnnotatedDataframe) and invoking other R/Bioconductor functions. The various modules for microarray data analysis were implemented only for 3' IVT Affymetrix cartridges and could be also customized by experienced users. Additionally, filtering of ES was limited to
MAS5 detection calls in order to eliminate data that are likely to be noisy. DEG identification, experimental design methods, and probe annotation strongly require the implementation of new and updated packages. AMDA 2.13 includes many important improvements: a new interface, application to additional platforms, quality control features, probeset filtering, new experimental design, DEG selection, and functional annotation.

The gWidget interface implemented for Affymetrix, Illumina, and Agilent platforms allows the definition of all possible settings in a user-friendly way (Figure 1A). The data analysis for Affymetrix starts from binary.CEL files and can be customized with different algorithms and options to be executed throughout the analysis; four separate workflows specialized for each platform are available (Figure 2). This part of the pipeline is based on the affy, Oligo, Agi $4 \times 44$ PreProcess, and lumi Bioconductor packages (14). The different pre-processing steps are platform-dependent and the user can choose various pre-processing methods. Illumina and Agilent platforms follow the same workflow as Affymetrix, but differ in the pre-processing steps. 
Table 1. Comparison of updated functionalities between AMDA and other well-known software.

\begin{tabular}{|c|c|c|c|c|c|}
\hline \multirow{2}{*}{$\begin{array}{l}\text { Updated Functionalities in AMDA } \\
\qquad 2.13\end{array}$} & \multicolumn{5}{|c|}{ Presence of the functionality in } \\
\hline & $\begin{array}{c}\text { GeneTrail } \\
\text { Express }\end{array}$ & GEPAS & GenePattern & Array Mining & OneChanneIGUI \\
\hline $\begin{array}{l}\text { 3' IVT .CEL pre-processing file } \\
\text { for cartridges and plates }\end{array}$ & - & Only for cartridges & - & - & Only for cartridges \\
\hline $\begin{array}{c}\text { Gene } 1.0 / 1.1 \text { ST and Exon } 1.0 \\
\text { ST .CEL } \\
\text { pre-processing }\end{array}$ & - & - & - & - & $\begin{array}{l}\text { Only Exon } 1.0 \mathrm{ST} \\
\text { (gene or exon level) }\end{array}$ \\
\hline $\begin{array}{c}\text { Agilent one-color } 4 \times 44 \text { pre- } \\
\text { processing }\end{array}$ & - & - & - & - & - \\
\hline 3' IVT call calculation & - & - & - & - & - \\
\hline Batch Correction (ComBat) & - & - & + & - & linear model batch effect \\
\hline Quality Controls (NUSE, RLE) & - & - & - & - & Only for 3' IVT \\
\hline $\begin{array}{c}\text { Prefiltering } \\
(I Q R, \text { annotation grade) }\end{array}$ & - & - & - & - & Only $D A B G$ and $I Q R$ \\
\hline $\begin{array}{l}\text { Illumina BeadChips } \\
\text { pre-processing }\end{array}$ & - & - & - & - & + \\
\hline $\begin{array}{l}\text { Methods for DEG selection } \\
\quad(\text { Limma, RP, SAM*) }\end{array}$ & $\begin{array}{l}\text { anova, Wilcoxon } \\
\text { Mann-Whitney and } \\
\text { Rank-Sum tests }\end{array}$ & $\begin{array}{l}\text { t-test, anova, clear } \\
\text { test }\end{array}$ & $\begin{array}{c}\text { Kolmogorov-Smirnov } \\
\text { test }\end{array}$ & $\begin{array}{l}\text { CFS, RF-MDA, } \\
\text { and PLS-CV } \\
(13)\end{array}$ & + \\
\hline $\begin{array}{c}\text { Support on experimental } \\
\text { Designs }\end{array}$ & - & - & - & - & Group comparison \\
\hline $\begin{array}{c}\text { Functional Evaluation (GO, } \\
\text { KEGG pathways, GSA) }\end{array}$ & $\begin{array}{c}\text { GSEA and } \\
\text { TRANSPATH (5) }\end{array}$ & $\begin{array}{l}\text { Not linked to } \\
\text { clusters selection }\end{array}$ & GSEA & + & Not linked to KEGG \\
\hline
\end{tabular}

Quality checks for arrays are performed through RLE and NUSE plots. RLE plots the deviation of each probeset from its median across all arrays, which, ideally, is 0 if most of the genes are unchanged. NUSE plots the standardized errors estimated from the Probe Level Model fit; so the median standard error across arrays is 1 for each gene.

PCA plots, which compare the three dimensions, are useful for quality control to identify outliers during pre-processing, as well as DEG variance for each group of replicates, and give an overview of the internal structure of the data set.

A more flexible data set-oriented filtering method using $I Q R$ function has been added for gene selection. The automatic choice of IQR implementation is based on its computational ease and ability to remove uninformative probesets that do not vary in the ES (Figure 3). Thus, signal-driven IQR gene filtering potentially reduces the number of false positives (Figure 3). The choice of probesets differs for Gene 1.0/1.1 ST, Exon 1.0 ST, and 3' IVT, which all depend on probe-matching position on the $\mathrm{mRNA}$ and genome annotation (Figure 1B).

The comparison between a common baseline and an experimental condition of a data set containing paired samples is performed through limma-paired exper- imental design and DEG selection. The experimental design can be defined with an appropriate text file for Affymetrix, Agilent, and Illumina platforms. The implementation of a paired-sample test makes AMDA 2.13 useful also for analyzing data from clinical studies (e.g., human lymphocytes before and after drug treatment).

Through addition of RP, AMDA 2.13 provides a straightforward and statistically meaningful way to determine DEG significance for two sample classes. The $\mathrm{RP}$ approach is not parametric, like Significance Analysis of Microarrays (SAM), and therefore requires less assumption on data distribution; it is powerful for both identifying biologically relevant expression changes and controlling the false discovery rate. It acts reliably in situations where very few replicates are available or in case of highly noisy data, by performing a permutation test on the set of replicates.

The implementation of novel algorithms for GO trimming eliminates local dependencies and points to relevant areas of the GO graph. The parent-child relationship, elim, and weight methods all address overlaps by computing statistical over-representation of DEG with counts weighted according to the hierarchical structure of the ontology.
Toward more meaningful knowledge through gene annotation, KEGG pathways were implemented for all platforms. DEG in KEGG gene sets that are mapped in the pathway diagram are enriched to assess the potential functional convergence of gene signatures based on KEGG pathway modules. DEG that are significantly enriched in a pathway are mapped on the KEGG graphical representation of the pathway as blue for down- and red for up-regulated genes. The topology of mapped DEG facilitates the selection of genes of interest in a specific pathway (Figure 4).

The evaluation of different gene sets through GSA could be useful to explore potentially interesting genes and give insight for biological interpretation of microarray data. If more than two conditions are assessed, GSA-positive and -negative gene score collections obtained are plotted through a heatmap, and gene sets can be observed. A negative score of one condition indicates lower expression of most genes in a gene set in comparison with higher values of the other condition; conversely, a positive score means that higher expression of most genes in the gene set correlates with higher values of the compared condition.

We have tested AMDA 2.13 on a microarray data set from a recent study (22), which was uploaded on the ArrayExpress Database (E-MEXP-2681). Total RNA 
extracted from muscle biopsies of patients with polymyositis (PM), dermatomyositis (DM), or juvenile myositis (JM), or of healthy individuals was compared using the commonBaseline design experiment. Test data set and report generated are available (Supplementary File 1). Quality assessment using RLE and NUSE showed that there were no obvious artifacts. Distance and variance between replicates are shown by hierarchical clustering and PCA plots. A total of 1,578 probesets were identified using the limma Bayesian statistical model (18), 876 of which distinguish controls from JM samples, 756 distinguish controls from DM, and 1,035 distinguish controls from PM. As indicated on the Venn diagram, a substantial number of genes showed differential expression in more than one comparison (Supplementary File 1). The results obtained are in full concordance with the findings reported in the original publication (22).

A comparison of the tools available in AMDA 2.13 with the functionalities provided by well-known softwares for microarray data analysis is shown in Table 1. OneChannelGUI (11), dChip (23), AltAnalyze (12), GenePattern (7), and GEPAS (4) offer a full set of tools for microarray data analysis comparable to those of AMDA. While crossplatform normalization pre-processing is provided by OneChannelGUI, AltAnalyze, and ArrayMining, these applications do not offer different experimental designs or a comprehensive output report that documents data in a workflow. With AMDA 2.13, the analytical modules can also be used separately by invoking the relative function as described (see Supplementary File 2). In addition, steps and results of the whole analysis are collectively described in a .pdf file report together with a set of .txt and .png files, thus improving the readability of global output.

AMDA 2.13 is not limited to analysis of data from the most commonly used commercial human, mouse, and rat arrays, but allows also the analysis of arrays for plant and yeast gene expression, such as $A$. thaliana and $S$. cerevisiae. It is implemented in $\mathrm{R}$ language in combination with Bioconductor, which makes it one of the most powerful and flexible command-driven solutions for microarray data analysis. It is also suitable for biologists lacking the necessary computational and statistical knowledge to address all aspects of a typical analysis workflow. In addition, the report gives a comprehensive explanation of the results obtained, together with brief explanations of each approach, and is particularly useful for biologists who are not familiar with programming concepts or statistical methodologies.

AMDA 2.13 is freely available as an $\mathrm{R}$ GPL package in the sourceforge.net (https:// sourceforge.net/projects/automicroarray/ files/) for Linux, Windows, and Mac OS operating systems. It has been tested on machines running Linux such as Debian GNU/Linux, Fedora, and OpenSUSE distributions, Windows 7, and Mac OS X 10.5.

\section{Acknowledgments}

We thank Drs. Matteo Barcella and Antonella Farinaccio for testing the software independently. This work was supported in part by TOLERAGE EC 7FP HEALTHF4-2008-202156 and FIGHT-MG EC 7FP HEALTH-F2-2010-242210.

\section{Competing interests}

The authors declare no competing interests.

\section{References}

1.Ashburner, M., C.A. Ball, J.A. Blake, D. Botstein, H. Butler, J.M. Cherry, A.P. Davis, K. Dolinski, et al. 2000. Gene ontology: tool for the unification of biology. The Gene Ontology Consortium. Nat. Genet. 25:25-29.

2. Ogata, H., S. Goto, K. Sato, W. Fujibuchi, H. Bono, and M. Kanehisa. 1999. KEGG: Kyoto Encyclopedia of Genes and Genomes. Nucleic Acids Res 27:29-34.

3. Pelizzola, M., N. Pavelka, M. Foti, and P. RicciardiCastagnoli. 2006. AMDA: an R package for the automated microarray data analysis. BMC Bioinformatics 7:335.

4. Tárraga, J., I. Medina, J. Carbonell, J. HuertaCepas, P. Minguez, E. Alloza, F. Al-Shahrour, S. Vegas-Azcárate, et al. 2008. GEPAS, a web-based tool for microarray data analysis and interpretation. Nucleic Acids Res. 36:W308-314.

5. Keller, A., C. Backes, M. Al-Awadhi, A. Gerasch, J. Küntzer, O. Kohlbacher, M. Kaufmann, and H.P. Lenhof. 2008. GeneTrailExpress: a web-based pipeline for the statistical evaluation of microarray experiments. BMC Bioinformatics 9:552.

6. Hull, D., K. Wolstencroft, R. Stevens, C. Goble, M.R. Pocock, P. Li, and T. Oinn. 2006. Taverna: a tool for building and running workflows of services. Nucleic Acids Res. 34:W729-732.

7. Reich, M., T. Liefeld, J. Gould, J. Lerner, P. Tamayo, and J.P. Mesirov. 2006. GenePattern 2.0. Nat. Genet. 38:500-501.

8. Giardine, B., C. Riemer, R.C. Hardison, R. Burhans, L. Elnitski, P. Shah, Y. Zhang, D. Blankenberg, et al. 2005. Galaxy: a platform for interactive large-scale genome analysis. Genome Res. 15:1451-1455.

9. Pan, F., K. Kamath, K. Zhang, S. Pulapura, A. Achar, J. Nunez-Iglesias, Y. Huang, X. Yan, et al. 2006. Integrative Array Analyzer: a software package for analysis of cross-platform and cross-species microarray data. Bioinformatics 22:1665-1667.

10. Kapushesky, M., P. Kemmeren, A.C. Culhane, S. Durinck, J. Ihmels, C. Körner, M. Kull, A. Torrente, et al. 2004. Expression Profiler: next generation-an online platform for analysis of microarray data. Nucleic Acids Res. 32:W465-470.

11. Sanges, R., F. Cordero, and R.A. Calogero. 2007. oneChannelGUI: a graphical interface to Bioconductor tools, designed for life scientists who are not familiar with $\mathrm{R}$ language. Bioinformatics 23:3406-3408.

12. Emig, D., N. Salomonis, J. Baumbach, T. Lengauer, B.R. Conklin, and M. Albrecht. 2010. AltAnalyze and DomainGraph: analyzing and visualizing exon expression data. Nucleic Acids Res. 38:W755-762.

13. Glaab, E., J.M. Garibaldi, and N. Krasnogor. 2009. ArrayMining: a modular web-application for microarray analysis combining ensemble and consensus methods with cross-study normalization. BMC Bioinformatics 10:358.

14. Gentleman, R.C., V.J. Carey, D.M. Bates, B. Bolstad, M. Dettling, S. Dudoit, B. Ellis, L. Gautier, et al. 2004. Bioconductor: open software development for computational biology and bioinformatics. Genome Biol. 5:R80.

15. Leek, J.T., R.B. Scharpf, H.C. Bravo, D. Simcha, B. Langmead, W.E. Johnson, D. Geman, K. Baggerly, and R.A. Irizarry. 2010. Tackling the widespread and critical impact of batch effects in high-throughput data. Nat. Rev. Genet. 11:733-739.

16. Johnson, W.E., C. Li, and A. Rabinovic. 2007. Adjusting batch effects in microarray expression data using empirical Bayes methods. Biostatistics 8:118-127.

17. Yeung, K.Y. and W.L. Ruzzo. 2001. Principal component analysis for clustering gene expression data. Bioinformatics 17:763-774.

18. Smyth, G.K. 2004. Linear models and empirical bayes methods for assessing differential expression in microarray experiments. Stat Appl Genet Mol Biol 3:Article3.

19. Breitling, R., P. Armengaud, A. Amtmann, and P. Herzyk. 2004. Rank products: a simple, yet powerful, new method to detect differentially regulated genes in replicated microarray experiments. FEBS Lett. 573:83-92.

20. Alexa, A., J. Rahnenfuhrer, and T. Lengauer. 2006. Improved scoring of functional groups from gene expression data by decorrelating GO graph structure. Bioinformatics 22:1600-1607.

21. Maglott, D.R., K.S. Katz, H. Sicotte, and K.D. Pruitt. 2000. NCBI's LocusLink and RefSeq. Nucleic Acids Res. 28:126-128.

22. Cappelletti, C., F. Baggi, F.Zolezzi, D. Biancolini, O. Beretta, M. Severa, E.M. Coccia, P. Confalonieri, et al. 2011. Type I interferon and Toll-like receptor expression characterizes inflammatory myopathies. Neurology 76:2079-2088.

23. Amin, S.B., P.K. Shah, A. Yan, S. Adamia, S. Minvielle, H. Avet-Loiseau, N.C. Munshi, and C. Li. 2011. The dChip survival analysis module for microarray data. BMC Bioinformatics 12:72.

Received 20 December 2011; accepted 04 June 2012.

Address correspondence to Dimos Kapetis, Fondazione IRCCS Istituto Neurologico Carlo Besta, Via Celoria 11, Milan 20133, Italy. Email: dimos.kapetis@istituto-besta.it

Contact Maria Foti for matters regarding Genopolis and the AMDA platform. Email: info@genopolis.it

To purchase reprints of this article, contact: biotechniques@fosterprinting.com 